\title{
Charge-Transfer Fluorescence Quenching by Electric Fields
}

\author{
Masaaki YokoYAmA, Akitoshi MAtSubARA, ${ }^{*}$ Shigeru ShimokiharA, \\ and Hiroshi MIKAWA
}

Department of Applied Chemistry, Faculty of Engineering, Osaka University, Yamadaoka, Suita, Osaka 565, Japan.

(Received July 16, 1981)

\begin{abstract}
KEY WORDS CT Fluorescence / Field-Induced Fluorescence Quenching / Sensitized Photocurrent / Carrier Photogeneration / Poly $(N$-vinylcarbazole) / 1,2,4,5-Tetracyanobenzene /
\end{abstract}

Field-induced charge transfer (CT) fluorescence quenching has been observed in a poly $(N$-vinylcarbazole) (PVCz) film doped with 1,2,4,5-tetracyanobenzene (4CNB), and this system was found to exhibit a large sensitized photocurrent in the corresponding $\mathrm{CT}$ absorption region. The $\mathrm{CT}$ fluorescence quenching is attributable to carrier photogeneration due to the field-assisted thermal dissociation of an ion pair which is produced from the excited CT state of the complex as a precursor. Analysis within the framework of the Onsager model for the dissociation process of an ion pair satisfactorily explains the experimental data of the field- and temperaturedependences of fluorescence quenching by electric fields.

The present CT complex sensitization system provides a favorable model of the famous $\mathrm{PVCz}-$ TNF (2,4,7-trinitrofluorenone) sensitized photoreceptor in the field of electrophotography. ${ }^{1,2}$ In previous papers, ${ }^{3,4}$ we reported that exciplex fluorescence observed in a $\mathrm{PVCz}$ film doped with relatively weak electron acceptors such as dimethyl terephthalate, for instance, is quenched by applying electric fields. This field-induced exciplex fluorescence quenching in the exciplex sensitization systems is attributable to the field-assisted thermal dissociation of ion pair produced from "nonrelaxed" exciplex state. Related to this exciplex sensitization, the carrier photogeneration in the CT complex system having charge-transfer interaction

* Present address: Konishiroku Photo Industry Co. Ltd., Hino, Tokyo 191, Japan. with stronger electron acceptor in the ground state is of great interest. The present CT complex system has the advantage in that an observable emission from its excited state as a precursor of ion pair gives valuable information on the charge photogeneration process.

When $4 \mathrm{CNB}$ is doped in a $\mathrm{PVCz}$ film, a CT absorption band appears at around $470 \mathrm{~nm}$, giving an orange colored film, and the CT fluorescence is observable at room temperature around $620 \mathrm{~nm}$ as shown in Figure 1.

Figure 2 shows the results of the steady-state photocurrent measurements. The sensitized photocurrent is clearly observed in the visible light region corresponding to the CT absorption band. Such an action spectrum of the photocurrent also gives good correspondence with the exciting wavelength dependence of field-induced CT fluorescence quenching, $\Delta F$ (dashed curve), shown in the same figure. These results strongly suggested that the excited state of CT complex plays an important role in the sensitization of carrier photogeneration.

The intensity of the $620 \mathrm{~nm}$ CT fluorescence decreased with the application of electric fields. The details of the fluorescence quenching experiments are the same as described in previous papers. ${ }^{3,4}$ Figure 3 shows the field-strength dependence of the field-induced fluorescence quenching ratio, $\Delta F / F$, of the decrement by applying a field, $\Delta F$, and the total fluorescence intensity in the absence of the field, $F$. The decrement of $\Delta F / F$ showed nearly the same values with respect to the excitation wavelengths, i.e., the excitations of the $\mathrm{CT}$ absorption band of the 


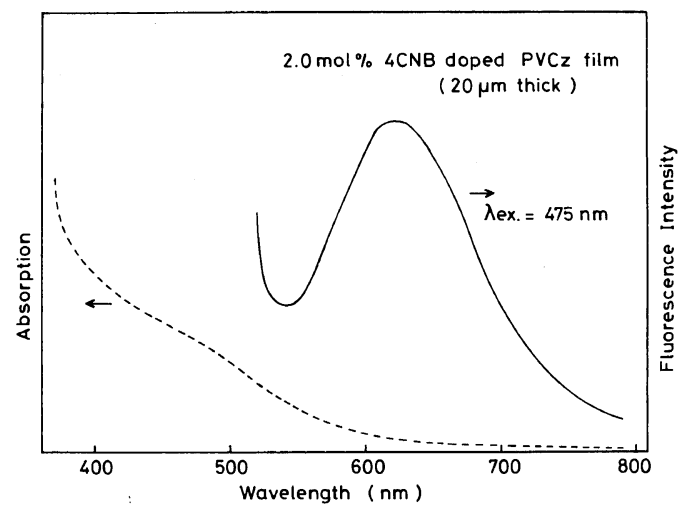

Figure 1. CT absorption and CT fluorescence spectra of a PVCz film doped with $2 \mathrm{~mol} \%$ of $4 \mathrm{CNB}$.

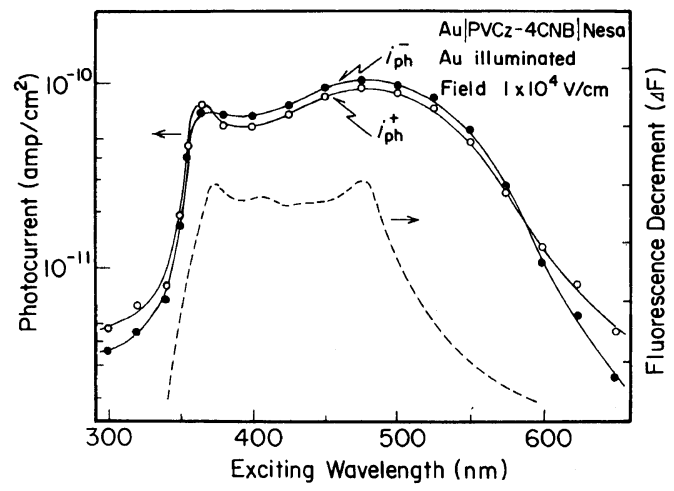

Figure 2. (a) Spectral dependence of steady-state photocurrents of $2.0 \mathrm{~mol} \% 4 \mathrm{CNB}$ doped $\mathrm{PVCz}$ film in a Au-Sample-Nesa sandwich-type cell. $i_{\mathrm{ph}}^{+}$and $i_{\mathrm{ph}}^{-}$: positive and negatively biased $\mathrm{Au}$ electrode illumination, respectively. Film thickness, $15 \mu \mathrm{m}$; field $=10^{4} \mathrm{~V} \mathrm{~cm}^{-1}$; $10^{12}$ photons $\mathrm{cm}^{-2}$; in high vacuum at room temperature. (b) Fluorescence decrement $\Delta F$ (dashed curve) by applying voltage $(300 \mathrm{~V})$ in the same cell assembly. Monitored in $620 \mathrm{~nm}$ CT fluorescence.

complex $(475,425 \mathrm{~nm})$ and carbazole $(\mathrm{Cz})$ chromophore $(365 \mathrm{~nm})$, and was nearly independent of the amount of the acceptors added. The decrement, $\Delta F / F$, increased with increasing applied fields. At a field strength of $2 \times 10^{5} \mathrm{~V} \mathrm{~cm}^{-1}$, for instance, the fluorescence decrement increases by nearly $3 \%$.

If the fluorescent process is competing with the dissociation process of an ion pair produced from a certain excited state of the CT complex, the following expression can be obtained for the fluorescence decrement, $\Delta F / F$, using the Onsager's formulation $^{5,6}$ for the dissociation probability of an ion

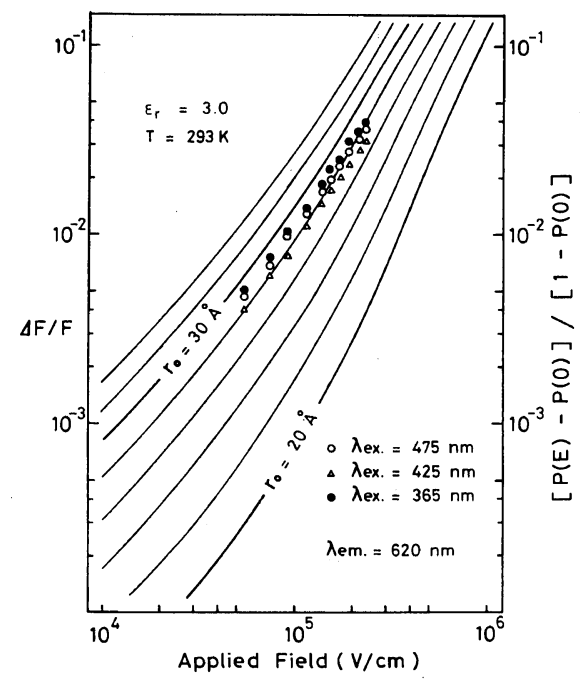

Figure 3. Field-strength dependence of field-induced CT fluorescence quenching in a $2.0 \mathrm{~mol} \%$ doped $\mathrm{PVCz}$ film at three excitation wavelengths in vacuum at $293 \mathrm{~K}$. This series of curves represents the theoretical prediction, $[P(E)-P(0)] /[1-P(0)]$ for various initial separations $r_{0}$ of ion pair.

pair, $P(E)$, at the initial separation $r_{0}$, as assumed in the case of previously reported exciplex fluorescence quenching. ${ }^{4}$

$$
\begin{aligned}
\Delta F / F & =[F(0)-F(E)] / F(0) \\
& =\eta \phi[1-P(0)]-\eta \phi[1-P(E)] / \eta \phi[1-P(0)] \\
& =[P(E)-P(0)] /[1-P(0)]
\end{aligned}
$$

Here, $F(E)$ denotes the fluorescence intensity in a field $E, \phi$ the quantum yield of the electron-hole pair, $\eta$ the fluorescence quantum efficiency. $P(E)$ can be calculated if the relative permittivity, $\varepsilon_{\mathrm{r}}$, temperature, $T$, and the initial separation of an ion pair, $r_{0}$, are known, as described in previous papers. ${ }^{4}$

The curves shown in Figure 3 represent the theoretical curves of $\Delta F / F$, i.e., $[P(E)-P(0)] /$ $[1-P(0)]$, for various initial separations $r_{0}$ with assuming $\varepsilon_{\mathrm{r}}=3.0$ at $293 \mathrm{~K}$. The experimental data gave good correspondence with the theoretical curve predicted for the ion pair of initial separation of $r_{0}=30 \AA$. In addition, a separate experiment for the temperature dependence of $\Delta F / F$ at a constant field also showed very good agreement between the experimental data and the theoretical curve of $r_{0}=$ $30 \AA$. The value of $r_{0}=30 \AA$ compares reason- 
ably well with the values obtained from photocurrent measurements, e.g., $30 \AA$ for the present $\mathrm{PVCz}-4 \mathrm{CNB}$ system ${ }^{7}$ and $35 \AA$ for the PVCz-TNF system. $^{8}$

Thus, we have obtained evidence that carrier photogeneration occurs by the field-assisted thermal dissociation of ion pair of $r_{0}=30 \AA$, produced from the nonrelaxed excited state of CT complex as proposed previously in the exciplex systems of PVCz and weak electron acceptors. The detailed mechanism of carrier photogeneration in the excited donor-acceptor systems and the nature of the nonrelaxed excited state of CT complex as a precursor of an ion pair will be discussed in the forthcoming paper.

\section{REFERENCES}

1. R. M. Schafert, IBM Res. Develop., 15, 75 (1971).

2. W. D. Gill, J. Appl. Phys., 43, 5033 (1972).

3. M. Yokoyama, Y. Endo, and H. Mikawa, Chem. Phys. Lett., 34, 597 (1975).

4. M. Yokoyama, Y. Endo, and H. Mikawa, Bull. Chem. Soc. Jpn., 49, 1538 (1976); J. Luminescence, 12/13, 865 (1976); J. Chem. Phys., 75, 3006 (1981).

5. L. Onsager, J. Chem. Phys., 2,599 (1934); Phys. Rev., 54, 554 (1938).

6. N. Geacintov and M. Pope, Proceedings of 3rd International Conference on Photoconductivity, E. M. Pell, Ed., Pergamon, Oxford, 1971, p 289.

7. S. Shimokihara, M. Fujino, H. Mikawa, and M. Yokoyama, to be published.

8. P. J. Meltz, J. Chem. Phys., 57, 1694 (1972). 